\title{
Defense Gene Expression and Phenotypic Changes of Rice (Oryza sativa L.) at the Reproductive Stage in Response to Whitebacked Planthopper (Sogatella furcifera Horvath) Infestation
}

\author{
T. VicheKA ${ }^{1}$, S. Yun ${ }^{1}$, A.F. Lindain ${ }^{2}$, M. Sopheareth ${ }^{3}$, I.K. Chung ${ }^{4}$ and K.M. Kim ${ }^{1 *}$ \\ ${ }^{1}$ School of Applied Biosciences, College of Agriculture \& Life Sciences, \\ Kyungpook National University, Daegu, 41566, Korea \\ 2705 Bonifacio St. San Jose City, Nueva Ecija, Philippines \\ ${ }^{3}$ Department of Agro-Industry, Ministry of Agriculture, Forestry and Fisheries, \\ 242, Norodom Blvd, Phnom Penh, Cambodia \\ ${ }^{4}$ Department of Biotechnology, Catholic University of Daegu, Gyeongsan-Si, Gyeongbuk, 38430, Korea \\ (Received 11 November 2016; Accepted 9 January 2017; \\ Communicated by J. Zhang)
}

This study was to determine the gene expression pattern and phenotypic change of Cheongcheong, Nagdong, TN1, and 8 different pedigrees of the CNDH population when WBPH infestation initiated at the reproductive stage of the crop. WBPH infested plants generally showed higher expression level of defense genes compared with the uninfected plants. $L O X$ transcriptional levels in Nagdong and CNDH42-1 did not increase after WBPH feeding at all-time course. Chlorophyll content declined in infested plants compared to their controls, but still CNDH3, CNDH14-2, and CNDH65 were healthier. Heavy and extensive WBPH feeding affected rice yield and grain quality although the infestation started at the reproductive stage.

Keywords: chlorophyll, rice, transcript, whitebacked planthopper

\section{Introduction}

With increasing demand due to human population growth, rice breeders have been developing many high-yielding and disease-resistant varieties to fulfill the global requirement. Rice plant is challenged by number of stresses, both biotic and abiotic, which can greatly affect the production and quality of grain. The whitebacked planthopper (WBPH, Sogatella furcifera Horvath) is one of the biotic stresses of rice plants that greatly affects rice yield and causes large economic losses (Yamasaki et al. 2003). These insects remove nutrients from the phloem sap and also transmit the southern rice black-streaked dwarf virus to the plant (Zhou et al. 2013). Plants trigger defense responses that are mediated through complex signal pathways in response to pathogen invasion, resulting to activation of defense genes. Phenylalanine ammonia-lyase (PAL) is the primary enzyme in the

\footnotetext{
*Corresponding author; E-mail: kkm@knu.ac.kr; Phone: +82-53-950-5711; Fax: +82-53-958-6880
} 
phenylpropanoid pathway, which is involved in biosynthesis of salicylic acid (MauchMani and Slusarenko 1996). Phenylpropanoids are derived from cinamic acid, which is formed from phenylalanine (Williams et al. 2005). Phenylpropanoids play essential roles in plant development and protection against environment stresses. A large number of studies reported that PAL expression is considered as plant defense which is responsible to abiotic and biotic stress such as pathogen attack, UV irradiation, wounding, and light (Chaman et al. 2003). Lipoxygenase (LOX) are nonheme iron-containing dioxygenases which broadly distribute in plants and animals. These enzymes use dioxygen molecules to catalyze the hydroperoxidation of polyunsaturated fatty acid which contains a $(1 \mathrm{z}, 4 \mathrm{z})$ pentadiene structure (Brash 1999). Product of LOX biosynthesis pathway such as traumatin, jasmonic acid, oxylipins and volatile aldehydes are supposed to play a crucial role in signal transduction of wound response, pathogen attack, and plant growth and development (Rosahl 1996). In resistant rice plant, the activity of OsLOX1 was increased during BPH (Niaparvata lugens) feeding while OsHI LOX were expressed strongly by the rice striped stem borer [(SSB), (Chilo suppressalis, Zhou et al. 2009)]. WRKY proteins are a recently identified class of DNA-binding proteins that recognize the TTGAC(C/T) W-box elements found in the promoters of a large number of plant defense-related genes. The WRKY transcription factors are one of the largest families of transcriptional regulators in plants. WRKY transcript factor consists of all living organism and plays as regulator of gene expression. Expression of a large number of WRKY genes is induced by pathogen (Lippok et al. 2007). Furthermore, the rice salicylic acid (SA) pathway branches into WRKY45-regulated and NPR1 homologue (NH1)/Oryza sativa neuropeptide receptors-1 (OsNPR1)-regulated sub-pathways and WRKY45 conferred strong resistant to both rice blast and leaf blight diseases when overexpressed in rice (Shimono et al. 2012). In the present study, the activation of the defense genes was studied to understand the level of expression following WBPH infestation at the reproductive stage. It is important to identify the rice resistance to $\mathrm{WBPH}$ in order to select the interesting rice lines and provide information on resistance and susceptibility of these experimental plants for further use in breeding. The qRT-PCR analysis was performed to detail the differences between gene expression in defense genes related to SA and jasmonic acid (JA)/ethylene (ET) mediated-defense pathways at different times following WBPH infestation in 11 rice varieties. Chlorophyll content and agronomic traits of experimental rice plants were likewise determined to assess the further effects of WBPH feeding.

\section{Materials and Methods}

\section{Plant materials}

In this study, Cheongcheong, Nagdong, Taichung Native 1 (TN1, susceptible check plant), and 8 different pedigrees of the Cheongcheong/Nagdong Double Haploid (CNDH) population were used. We had 8 different pedigrees by coincident rate of quantitative trait loci (QTL) markers from comparison with phenotype and genotype of resistant and susceptible lines among $120 \mathrm{CNDN}$ lines (Yun et al. 2016). CNDH population belongs to the 
eight filial generations $\left(\mathrm{F}_{8}\right)$, which were derived from crossings between Cheongcheong and Nagdong. The Cheongcheong was moderately resistant, whereas Nagdong was moderately susceptible to WBPH (Kim et al. 2013). Seeds for these varieties were donated by the Kyungpook National University, Korea in 2013. Germinated seeds were sown in the greenhouse for 21 days, after which they were transplanted singly in plastic pots $(14 \mathrm{~cm}$ diameter $\times 24 \mathrm{~cm}$ tall). Plants were maintained under controlled temperature in a greenhouse according to the seasons until the reproductive stage. At this stage, the control and treatment plants were separately placed inside a transparent plastic net cage to prevent insect migration. Fertilizer was applied twice (during the $3^{\text {rd }}$ and $10^{\text {th }}$ week) after transplanting.

\section{WBPH population and rearing}

The WBPH population was provided by the National Institute of Crop Science (NICS), Rural Development Administration, Korea. The insectarium was maintained at $27 \pm 1{ }^{\circ} \mathrm{C}$, with a relative humidity of 60-70\%, and light cycle of 16-h light: 8-h dark. For breeding, adult WBPH were selected and transferred to rice seedlings, which were placed in plastic cages so the insects would produce the next generation homogeneously. Rice seedlings were replaced once a week. The WBPH populations were allowed to reproduce for several generations to confirm that they were the same stage of adults before being transferred into the rice plants.

\section{WBPH infestation}

Approximately 200 adult WBPHs were placed on each plant. Insects for use in the experiment were selected and maintained on the susceptible variety of seedling and then released on the treatment plant gently and evenly. Rice leaves were cut to analyze the level of gene expression of OsWRKY45 and NH1 at 0,30, 60, 90, and 120 min as minutes post infestation (mpi). Gene expression of $L O X$ and $P A L$ was also conducted at $0,24,48$, and 72 hours as hours post infestation (hpi). All samples were collected from the leaf blade, immediately frozen in liquid nitrogen, and stored at $-70{ }^{\circ} \mathrm{C}$ until use.

\section{Quantitative Real-time PCR (qRT-PCR) analysis of defense-related genes}

Total RNA from rice leaves was isolated using RNeasy Plant Mini Kit (QIAGEN, Germany). cDNA library was synthesized from total RNA using PhileKorea Kit. Quantitative Eco Real Time PCR (Illumina, USA) was used in this study. The PCR primer pairs in PAL (GGTGTTCTGCGAGGTGATGA, AGGGTGCTTCAGCTTGT), LOX (CATGGAAGTCGAGCATGAACA, AATAAAGATTTGGGAGTGACATA), WRKY 45 (TCGTCAAGAACCTCGACGAC, TGTCATCTCAGCGGCTTCC), NH1 (TGGCAGGTGAGAGTCTACGA, TCAGGAGGTGGATTTGCACC), and OsActin (ATCCTTGTATGCTAGCGGTCGA, ATCCAACCGGAGGATAGCATG) were used to amplify the genes of interest in this study. The qRT-PCR conditions were $95^{\circ} \mathrm{C}$ for 2 minutes for the activa- 
tion of the polymerase, followed by 40 cycles at $95{ }^{\circ} \mathrm{C}$ for $10 \mathrm{~s}, 60^{\circ} \mathrm{C}$ for $30 \mathrm{~s}$, and $72{ }^{\circ} \mathrm{C}$ for $15 \mathrm{~s}$, and a final melting curve at $95^{\circ} \mathrm{C}$ for $15 \mathrm{~s}, 55^{\circ} \mathrm{C}$ for $15 \mathrm{~s}$, and $95^{\circ} \mathrm{C}$ for $15 \mathrm{~s}$. A chlorophyll meter (SPAD 502 Minolta Camera Co. Ltd., Japan) was used to determine chlorophyll content after 1, 3, 5, 7, and 9 weeks post infestation during the reproductive stage of rice. Triplicate readings were done from the middle area of the flag leaf of each plant and averaged.

\section{Assessment of yield and yield components and statistical analysis}

The yield of treatment and control plants were determined and compared. Yield assessment based on spikelet number per panicle, 100-seed weight and percentage of productive seeds per plant were also obtained. Yield loss was computed using the following formula: $\mathrm{L}=\mathrm{Y}_{\mathrm{p}}-\mathrm{Y}_{\mathrm{o}} / \mathrm{Y}_{\mathrm{p}} \times 100(\mathrm{~L}=$ Percentage of yield loss due to $\mathrm{WBPH}$ infestation, $\mathrm{Y}_{\mathrm{p}}=$ Yield of uninfested plants, $\mathrm{Y}_{\mathrm{o}}=$ Yield of infested plants). The experiment was completely randomized block design with three replicates per treatment. Phenotypic data analyzed statistically by using the SAS program (ver. 9.4).

\section{Results}

Results in Fig. 1 show the expression pattern of 4 defense-related genes (PAL, LOX, $W R K Y 45$, and $N H 1$ ) in 11 rice varieties/lines tested in this study in response to WBPH infestation at reproductive stage. Generally, all plants infested with WBPH showed higher expression levels of defense genes compared with the uninfested plants (control). $P A L$ is the major gene related to the SA-synthesis pathway. The basal transcript levels of $P A L$ were slightly different in all plants. By $24 \mathrm{hpi}$, the transcript level of $P A L$ was only induced in Cheongcheong, Nagdong, CNDH14-2, CNDH31, CNDH65, and CNDH92-2. Moreover, PAL levels decreased in CNDH42-2 and CNDH45; whereas that of CNDH3 and CNDH31 remained similar to the basal levels. Remarkably, at 24 hpi, PAL level decreased in majority of the plants, but CNDH65 had the highest expression values among them. By 48 hpi, the expression of PAL decreased in all plants except for CNDH3 which remained constant. By 72 hpi, the transcript level of Cheongcheong, CNDH3, CNDH31, CNDH42-1, CNDH42-2, CNDH45, CNDH65, and CNDH92-2 again increased, but PAL transcript level slightly decreased in CNDH14-2. In addition, the expression level in Nagdong remained similar at 48 and 72 hpi. LOX expression values in all plants were different at their basal level. By $24 \mathrm{hpi}$, the level of expression of $L O X$ decreased in all treatment plants except in CNDH92-2, wherein the expression was slightly induced. It was observed that there was a decrease in the expression level of $L O X$ from 0 to 24 hpi in CNDH31. CNDH45 and CNDH65, but at 48 hpi the expression again increased, wherein the transcript level of CNDH65 was the highest. On the other hand, LOX level at 48 hpi in the remaining test plants declined again, but at $72 \mathrm{hpi}$, level increased again except for Nagdong, CNDH42-1, CNDH45, CNDH65 and CNDH92-2. By 72 hpi, LOX levels in CNDH3 and CNDH14-2 were significantly higher than that of the resistant Cheongcheong. It is also important to note that $L O X$ transcript levels in Nagdong and CNDH42-1 did not 

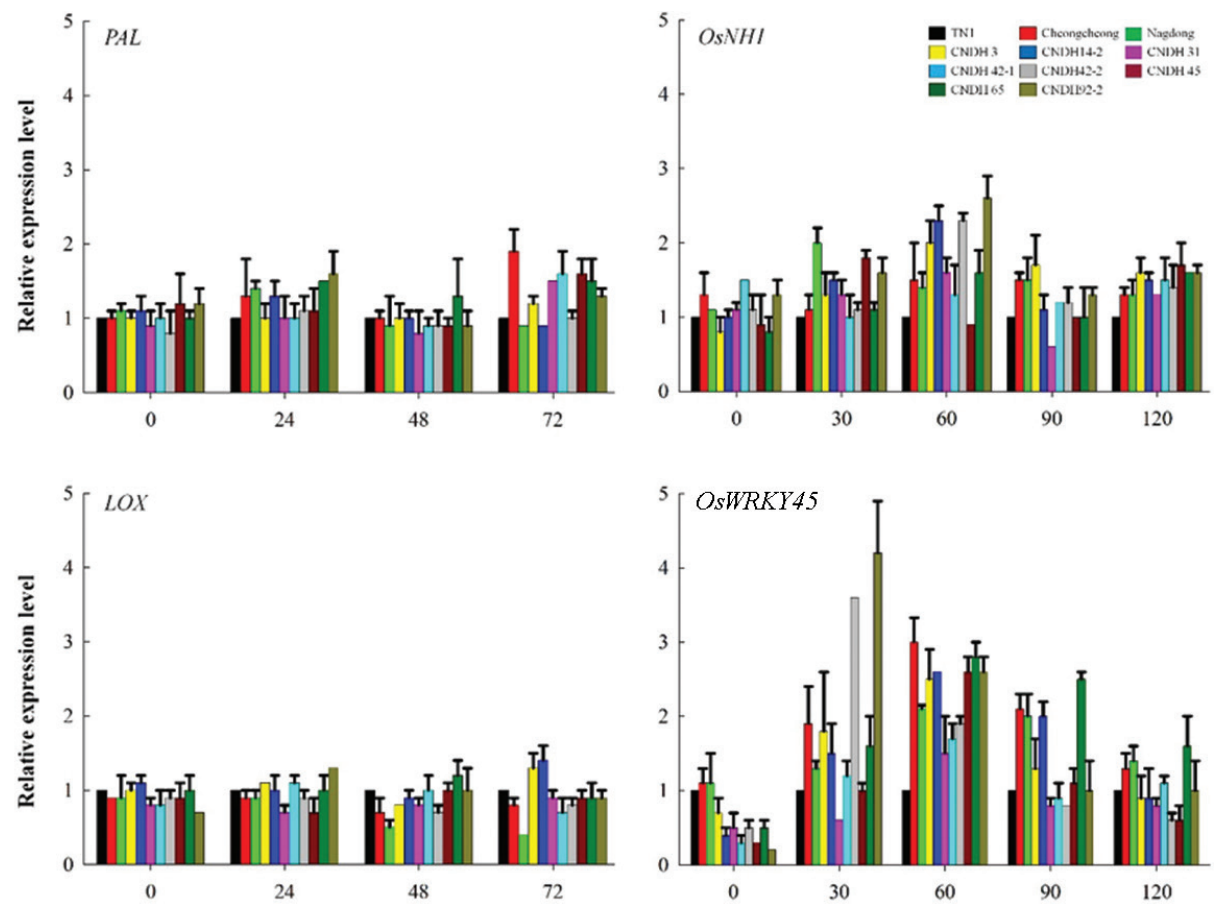

Figure 1. The induced expression of defense genes in TN1, Cheongcheong, Nagdong and 8 different pedigree (CNDH3, CNDH14-2, CNDH31, CNDH42-1, CNDH42-2, CNDH45, CNDH65, CNDH92-2) among CNDH population upon WBPH infestation at (0), 24, 48, and $72 \mathrm{~h}$ for $P A L$ and $L O X$ and (0), 30, 60, 90, and $120 \mathrm{~min}$ for OsWRKY45 and OsNH1 using qRT-PCR. PAL and transcription factor WRKY45 are genes involved in SA-synthesis pathway. NH1 is a crucial regulatory gene in SA-dependent systemic acquired resistance. LOX is JA synthetic-related gene

increase in response to $\mathrm{WBPH}$ at all-time course. WRKY transcription factors play an important role in regulating both biotic and abiotic stresses in plants. To identify the function of rice $W R K Y$ genes following the WBPH infestation, the expression of the OsWRKY45 gene was determined using qRT-PCR. The expression showed that the transcript level of WRKY45 was induced in all plants at $30 \mathrm{mpi}$ following WBPH feeding. The transcript level in all plants increased from 30 to 60 mpi, excluding that of CNDH 42-2 and CNDH92-2, in which levels decreased at 60 mpi and declined smoothly from 60 to 90 mpi until a low level at 120 mpi was maintained. Remarkably, the transcript level of OsWRKY45 was highest in CNDH92-2 and followed by CNDH42-2 at $30 \mathrm{mpi}$. The expression analysis indicated that the $O s W R K Y$ gene in all rice plants was associated with the defense response to WBPH infestation, in particular, OsWRKY gene functioned at 30 and $60 \mathrm{mpi}$ in the defense signaling pathway. The transcript level of $\mathrm{NH} 1$ in all plants was similarly expressed at $0 \mathrm{mpi}$, but level declined at $30 \mathrm{mpi}$, excluding Nagdong in which it was slightly increased in response to WBPH feeding. By 60 mpi, 4 (CNDH3, CNDH14-2, CNDH42-2, and CNDH92-2) of the 11 lines had transcript levels that rose substantially 


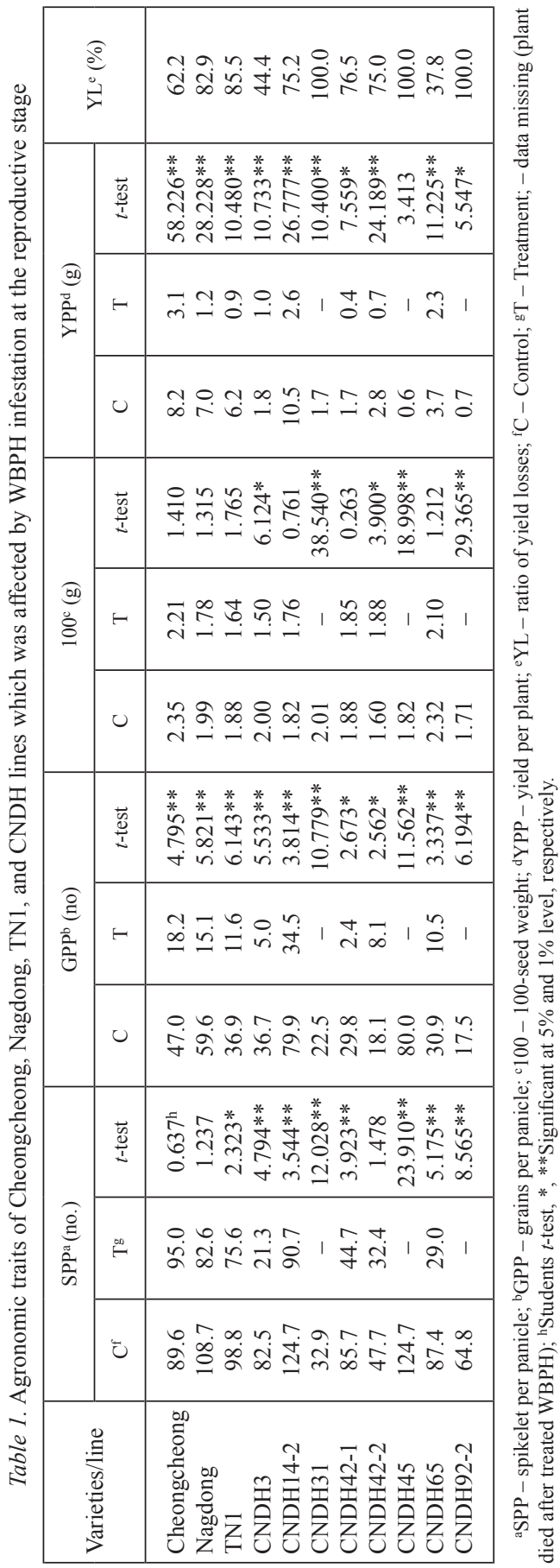

Cereal Research Communications 45, 2017 
compared to the resistant Cheongcheong, whereas others were maintained a steady transcript expression following insect infestation. The expression level of the NHI gene smoothly declined at $90 \mathrm{mpi}$ and was maintained until $120 \mathrm{mpi}$. The activation of $\mathrm{NH}$ may be involved in regulation of SA in response to WBPH infestation in subsequent time point.

Chlorophyll was measured after every two weeks post infestation. Plants were fed on by a large number of planthoppers which resulted to decreased chlorophyll content of plants and some eventually died (CNDH31, CNDH45, and CNDH92-2). The SPAD values for rice leaves are presented in Fig. S1*. Significant differences in SPAD values occurred between the rice plants infested by WBPH and the controls.

The effect of WBPH infestation on plants at the reproductive stage is generally complex and variable. Yield loss caused by infestation was observed in rice plants at the reproductive stage following feeding by an estimated $200 \mathrm{WBPH}$ per 10 plants. Yield loss due to WBPH feeding on the 11 rice plant varieties was dramatic at $37.8-100 \%$ decline. The losses varied among the tested varieties. Completed loss of yield occurred in CNDH31, CNDH45, and CNDH92-2. Similarly, CNDH14-2, CNDH42-1 and CNDH422 showed the high losses, which were 75.2, 76.5, and 75.0\%, respectively. Remarkably, CNDH3 and CNDH65 seemed to be resistant to WBPH infestation in comparison to the resistant Cheongcheong, in which yield losses were 44.4 and $37.8 \%$, respectively. The number of spikelet per panicle greatly varied (29-95), as did the number of fertile grains per panicle, which was $0.0-53.4 \%$ (data not shown). The lowest spikelet number $(0.0)$ was recorded for CNDH31, CNDH45 and CNDH92-2, and the highest (90.7) for CNDH14-2. The highest 100-seed weight (2.10 g) was obtained from CNDH 65, whereas the control reference was $2.35 \mathrm{~g}$. The highest significant yield per plant $(2.6 \mathrm{~g})$ occurred for CNDH14-2 and the control reference was $10.5 \mathrm{~g}$ (Table 1).

\section{Discussion}

The whitebacked planthopper is one of the most damaging pests that ingest the phloem sap of rice. Development of resistant rice varieties is one of the methods to minimize damage caused by the planthopper and other herbivores. Rice varieties could be bred with resistant $(\mathrm{R})$ genes for resistance to planthoppers, or a combination of genes for resistance against numerous pests. In this study, WBPH-responsive genes PAL, LOX, OsWRKY45, and $O s N H 1$ were analyzed to characterize rice cultivars. These defensive genes are regulated by signaling pathways related to regulators JA, ET, and SA (Lyons et al. 2013). It is believed that the SA-mediated signaling pathway was activated by WBPH infestation and $P A L$ plays a crucial role in stimulating the signal pathway. The transcript level of the SA synthesis-involved gene $P A L$ was significantly higher in the resistant rice plant than in the susceptible variety in response to small brown planthopper infestation (Duan et al. 2014). The accumulation of $L O X$ gene related to the JA/ET signaling pathway was greater in CNDH3, CNDH14-2, CNDH65, and CNDH92-2 than the parent plants, Cheongcheong and Nagdong. Similarly, an escalation in $L O X$ has been reported to occur in tomato plants

*Further details about the Electronic Supplementary Material (ESM) can be found at the end of the article. 
after infection (Koch et al. 1992). Our data indicate that $L O X$ would participate in defending against WBPH in CNDH3, CNDH14-2, CNDH65, and CNDH92-2 and may account for their resistance. Inoculation of the avocado (Persea americana) plant with Phytophthora cinnamomi caused differences in transcript levels of $L O X$, which may suggest that the expression of this gene contributes to resistance (Engelbrecht and Van den Berg 2013). In the present study, the expression of gene defense at the basal level was lower during pre-infestation, but immediately increased following WBPH exposure. Therefore, the expression level could indicate that OsWRKY45 was activated earlier in defense response to WBPH infestation in all rice plants, although the degree of expression was slightly different. OsWRKY45 plays a crucial role in the response to WBPH infestation in all rice plants. For instance, constitutive overexpression of OsWRKY45 in transgenic rice plants enhanced resistance to rice blast fungus (Shimono et al. 2012). Goto et al. (2015) reported that overexpression of OsWRKY45 could affect the tolerance of rice plants to low temperatures. Overexpression of $O s N H 1$ indicated a boost in resistance of the rice plant after infestation. Expression of $P A L, O s N H 1$, and $O s W R K Y 45$ is related with the activation of the SA signaling pathway, whereas $L O X$ is involved in JA biosynthesis (Ogawa et al. 2005). It is suggested that defense gene of rice against WBPH were activated in CNDH3, CNDH14-2, CNDH42-2, and CNDH92-2. The capacity for photosynthesis of rice leaves is related to nitrogen content because the proteins in the Calvin cycle and thylakoids represent the majority of leaf nitrogen (Evans 1989). Most WBPH damage is on the main stem of the rice plant where the phloem sap contains sucrose and nitrogen compounds (Kehr 2006). The removal of these nutrients affects the growth and development of the plant (Rubia-Sanchez et al. 1999), and eventually leads to death. The physiology and biochemistry of rice plants were altered after infestation because rice phloem is a major mediator in plant communication (Ruiz-Medrano et al. 2001). SPAD value changed in the rice plant after infestation due to the chlorophyll degradation in the chloroplast. Furthermore, BPH feed and grow on either susceptible or resistant plants at the flowering stage, although the plant expressed resistance at the vegetative stage (Jairin et al. 2007). Pathogen infection usually causes a significant decrease in photosynthesis and in turn decreases the availability of nutrients for the pathogen (Berger et al. 2007). The infection leads to changes in secondary metabolism based on the induction of defensive systems together with primary metabolism that affects the development and yield of the plant. The yield and weight of susceptible rice plants was lost when the chlorophyll content was decreased because of heavy infestation of WBPH. These results support insight the expression patterns of defense-related genes against insect attack and assist the development of resistance varieties to prevent this destructive insect.

\section{Acknowledgements}

The authors are grateful for the financial assistance granted by the Next-Generation BioGreen21 Program (No. PJ011257012017), Rural Development Administration, Republic of Korea for this research. This research was supported by the Kyungpook National University Research Fund, 2016. 


\section{References}

Berger, S., Sinha, A.K., Roitsch, T. 2007. Plant physiology meets phytopathology: plant primary metabolism and plant-pathogen interactions. J. Exp. Bot. 58:4019-4026.

Brash, A.R. 1999. Lipoxygenases: occurrence, functions, catalysis, and acquisition of substrate. J. Biol. Chem. 274:23679-23682.

Chaman, M.E., Copaja, S.V., Argandoña, V.H. 2003. Relationships between salicylic acid content, phenylalanine ammonia-lyase (PAL) activity, and resistance of barley to aphid infestation. J. Agric. Food Chem. 51:2227-2231.

Duan, C., Yu, J., Bai, J., Zhu, Z., Wang, X. 2014. Induced defense responses in rice plants against small brown planthopper infestation. Crop J. 2:55-62.

Engelbrecht, J., Van den Berg, N. 2013. Expression of defense-related genes against Phytophthora cinnamomi in five avocado rootstocks. S. Afr. J. Sci. 109:1-8.

Evans, J.R. 1989. Photosynthesis and nitrogen relationships in leaves of C3 plants. Oecologia 78:9-19.

Jairin, J., Teangdeerith, S.N., Leelagud, P., Phengrat, K., Vanavichit, A., Toojinda, T. 2007. Physical mapping of Bph3, a brown planthopper resistance locus in rice. Maejo. Int. J. Sci. Technol. 1:166-177.

Goto, S., Sasakura-Shimoda, F.M., Suetsugu, M., Selvaraj, G., Hayashi, N., Yamazaki, M., Takatsuji, H. 2015. Development of disease-resistant rice by optimized expression of WRKY45. Plant Biotechnol. J. 13:753765.

Kehr, J. 2006. Phloem sap proteins: their identities and potential roles in the interaction between plants and phloem-feeding insects. J Exp. Bot. 57:767-774.

Kim, T.H., Kim, K.M., Manigbas, N.L., Yi, G., Sohn, J.K. 2013. Identification of quantitative trait loci for resistance to white-backed planthopper (Sogatella furcifera) in rice with Milyang 46 (Cheongcheongbyeo) background. Philipp. J. Crop Sci. 38:30-36.

Koch, E., Meier, B.M., Eiben, H.G., Slusarenko, A. 1992. A lipoxygenase from leaves of tomato (Lycopersicon esculentum Mill.) is induced in response to plant pathogenic pseudomonads. Plant Physiol. 99:571-576.

Lippok, B., Birkenbihl, R.P., Rivory, G., Brümmer, J., Schmelzer, E., Logemann, E., Somssich, I.E. 2007. Expression of AtWRKY33 encoding a pathogen- or PAMP-responsive WRKY transcription factor is regulated by a composite DNA motif containing W box elements. Mol. Plant-Microbe Interact. 20:420-429.

Lyons, R., Manners, J.M., Kazan, K. 2013. Jasmonate biosynthesis and signaling in monocots: a comparative overview. Plant Cell Rep. 32:815-827.

Mauch-Mani, B., Slusarenko, A.J. 1996. Production of salicylic acid precursors is a major function of phenylalanine ammonia-lyase in the resistance of Arabidopsis to Peronospora parasitica. The Plant Cell 8:203212.

Ogawa, D., Nakajima, N., Sano, T., Tamaoki, M., Aono, M., Kubo, A., Saji, H. 2005. Salicylic acid accumulation under $\mathrm{O}_{3}$ exposure is regulated by ethylene in tobacco plants. Plant Cell Physiol. 46:1062-1072.

Rosahl, S. 1996. Lipoxygenases in plants - their role in development and stress response. Z. Nat. forsch. C. 51:123-138.

Rubia-Sanchez, E., Suzuki, Y., Miyamoto, K., Watanabe, T. 1999. The potential for compensation of the effects of the brown planthopper Nilaparvata lugens Stal (Homoptera: Delphacidae) feeding on rice. Crop. Prot. 18:39-45.

Ruiz-Medrano, R., Xoconostle-Cázares, B., Lucas, W.J. 2001. The phloem as a conduit for inter-organ communication. Curr. Opin. Plant Biol. 4:202-209.

Shimono, M., Koga, H., Akagi, A.Y.A., Hayashi, N., Goto, S., Sawada, M., Takatsuji, H. 2012. Rice WRKY45 plays important roles in fungal and bacterial disease resistance. Mol. Plant Pathol. 13:83-94.

Williams, J.S., Thomas, M., Clarke, D.J. 2005. The gene stlA encodes a phenylalanine ammonia-lyase that is involved in the production of a stilbene antibiotic in Photorhabdus luminescens TT01. Microbiol. 151:2543-2550.

Yamasaki, M., Yoshimura, A., Yasui, H. 2003. Genetic basis of ovicidal response to whitebacked planthopper (Sogatella furcifera Horvath) in rice (Oryza sativa L.). Mol. Breed. 12:133-143.

Yun, S., Lee, G.H., Kim, K.M. 2016. Optimum screening time for improved WBPH-associated QTL analysis in rice. Int. J. Agric. Biol. 18:844-850. 
Zhou, G., Qi, J., Ren, N., Cheng, J., Erb, M., Mao, B., Lou, Y. 2009. Silencing OsHI-LOX makes rice more susceptible to chewing herbivores, but enhances resistance to a phloem feeder. The Plant J. 60:638-648.

Zhou, G., Xu, D., Xu, D., Zhang, M. 2013. Southern rice black-streaked dwarf virus: a white-backed planthopper-transmitted fijivirus threatening rice production in Asia. Front Microbiol. 4:270.

\section{Electronic Supplementary Material (ESM)}

Electronic Supplementary Material (ESM) associated with this article can be found at the website of CRC at http://www.akademiai.com/content/120427/

Electronic Supplementary Figure S1. The SPAD reading (mean \pm SD) of each infested and uninfested rice plant at week $1,3,5,7$, and 9 post infestation during the late productive stage. A: TN1, B: Cheongcheong, C: Nagdong, D: CNDH3, E: CNDH14-2, F: CNDH31, G: CNDH42-1, H: CNDH42-2, I: CNDH45, J: CNDH65, K: CNDH92-2 